\title{
GRAVITATIONAL LENSING AS A POSSIBLE EXPLANATION FOR SOME UNIDENTIFIED GAMMA-RAY SOURCES AT HIGH LATITUDES
}

\author{
Diego F. Torres, ${ }^{1}$ Gustavo E. Romero, ${ }^{2}$ and Ernesto F. Eiroa ${ }^{3}$ \\ Received 2001 October 19; accepted 2001 December 26
}

\begin{abstract}
We propose that some of the high-latitude unidentified EGRET $\gamma$-ray sources could be the result of gravitational lensing amplification of the innermost regions of distant, faint, active galactic nuclei. These objects have $\gamma$-ray-emitting regions small enough to be affected by microlensing of stars in interposed galaxies. We compute the gravitational amplification, taking into account effects of the host galaxy of the lens, and prove that, whereas the innermost $\gamma$-ray regions can be magnified up to a thousand times, there is no amplification at radio frequencies, which leads to the observed absence of strong counterparts. Some new effects in the spectral evolution of a gravitational microlensed $\gamma$-ray active galactic nuclei are predicted. Within a reasonable range of lensing parameters and/or types of sources, both variable and nonvariable EGRET detections at high latitudes can be explained by microlensing. The same phenomenon could also have an important incidence among the future GLAST detections at high latitudes.
\end{abstract}

Subject headings: galaxies: active — gamma rays: observations — gravitational lensing

\section{INTRODUCTION}

The Third EGRET Catalog of $\gamma$-ray sources includes observations carried out between 1991 April 22 and 1995 October 3 and lists 271 pointlike detections (Hartman et al. 1999). About two-thirds of them have no conclusive counterparts at lower frequencies and are dubbed unidentified.

These unidentified $\gamma$-ray sources can be divided in two broad groups. The first one, at low latitudes, is probably related to several galactic populations such as radio-quiet pulsars, interacting supernova remnants, early-type stars with strong stellar winds, X-ray binaries, etc. (see Romero 2001 and references therein). The second group of unidentified sources is formed by mid- and high-latitude detections. Gehrels et al. (2000) have shown-in a model-independent way - that the mid-latitude sources are different from the bright population of unidentified sources along the Galactic plane. Some of the mid-latitude detections $\left(5^{\circ}<|b|<30^{\circ}\right)$ are thought to be associated with the Gould Belt (Grenier 2000; Gehrels et al. 2000), which is a starburst region lying $\sim 600 \mathrm{pc}$ from Earth. A few other sources at higher latitudes might be the result of electrons being accelerated at the shock waves of forming clusters of galaxies (Totani \& Kitayama 2002). However, for most of the high-latitude unidentified sources (HL-UnidS), no other explanation seems to be available than that they are active galactic nuclei (AGNs) yet unnoticed at lower energies (Reimer \& Bertsch 2001).

A $\gamma$-ray AGN population consisting of 66 members has already been detected by EGRET (Hartman et al. 1999). Photon spectra and variability indices of the HL-UnidS match well those of known $\gamma$-ray AGNs. In Figure 1, we show the corresponding distributions with the AGN histo-

\footnotetext{
${ }^{1}$ Department of Physics, Joseph Henry Laboratories, Jadwin Hall, P.O. Box 708, Princeton University, Princeton, NJ 08544-0708; dtorres@ princeton.edu.

${ }^{2}$ Instituto Argentino de Radioastronomía (IAR), Casilla de Correo Number 5, Villa Elisa-Provinica, 1894 Buenos Aires, Argentina; romero@irma.iar.unlp.edu.ar. Member of CONICET.

${ }^{3}$ Instituto de Astronomía y Física del Espacio, Casilla de Correo 67, Sucursal 28, 1428 Buenos Aires, Argentina; eiroa@iafe.uba.ar.
}

gram adapted from Torres et al. (2001a). The variability criterion used here is the $I$-scheme, but other variability criteria yield similar results (Torres, Pessah, \& Romero 2001b). Population studies have already remarked that part of the sample of HL-UnidS is consistent with an isotropic population, a fact that also supports an extragalactic origin (Özel \& Thompson 1996).

All identified $\gamma$-ray AGNs are also strong radio sources with flat spectrum, as expected from synchrotron jetlike sources where the $\gamma$-ray flux is the result of inverse Compton scattering (Mattox et al. 1997). We could ask, then, why the HL-UnidS are not detected at lower frequencies, particularly in the radio band, if they are also AGNs? Here, we propose that some of these sources are the result of gravitational lensing amplification of background, highredshift, active galactic nuclei, blazars whose $\gamma$-ray-emitting regions are small enough to be affected by microlensing by stars in interposed galaxies.

\section{GRAVITATIONAL LENSING OF ACTIVE GALACTIC NUCLEI}

Since AGNs have emission regions of different sizes for different wavelengths, we expect a differential magnification of light. The innermost regions of AGNs, responsible for the $\gamma$-ray emission, have linear sizes $x \sim 10^{14}-10^{15} \mathrm{~cm}$ (e.g., Blandford \& Levinson 1995). Significant gravitational lensing requires that $D_{o l} / D_{o s} \times x / 2<R_{\mathrm{E}}$, where $D_{o l}$ and $D_{o s}$ are the angular diameter distances between the lens $(l)$, or the source $(s)$, and the observer $(o)$, and $R_{\mathrm{E}}$ is the Einstein radius of the lens. For typical redshifts (say, $z$ approximately a few tenths for the lens, and $z \sim 0.8-3$ for the source), in a standard cosmological model, we find that stars of few solar masses have Einstein radii $~ 500$ times bigger than the projected AGN's $\gamma$-ray-emitting region and, thus, that the latter can be perfectly amplified. At the same time, since radio emission is originated far down the jet, the sizes of the corresponding radio regions $\left(x>10^{17} \mathrm{~cm}\right)$ exceed the Einstein radius of the lenses and lead to the absence of radio counterparts. In the case of optical emission, if it is the same 

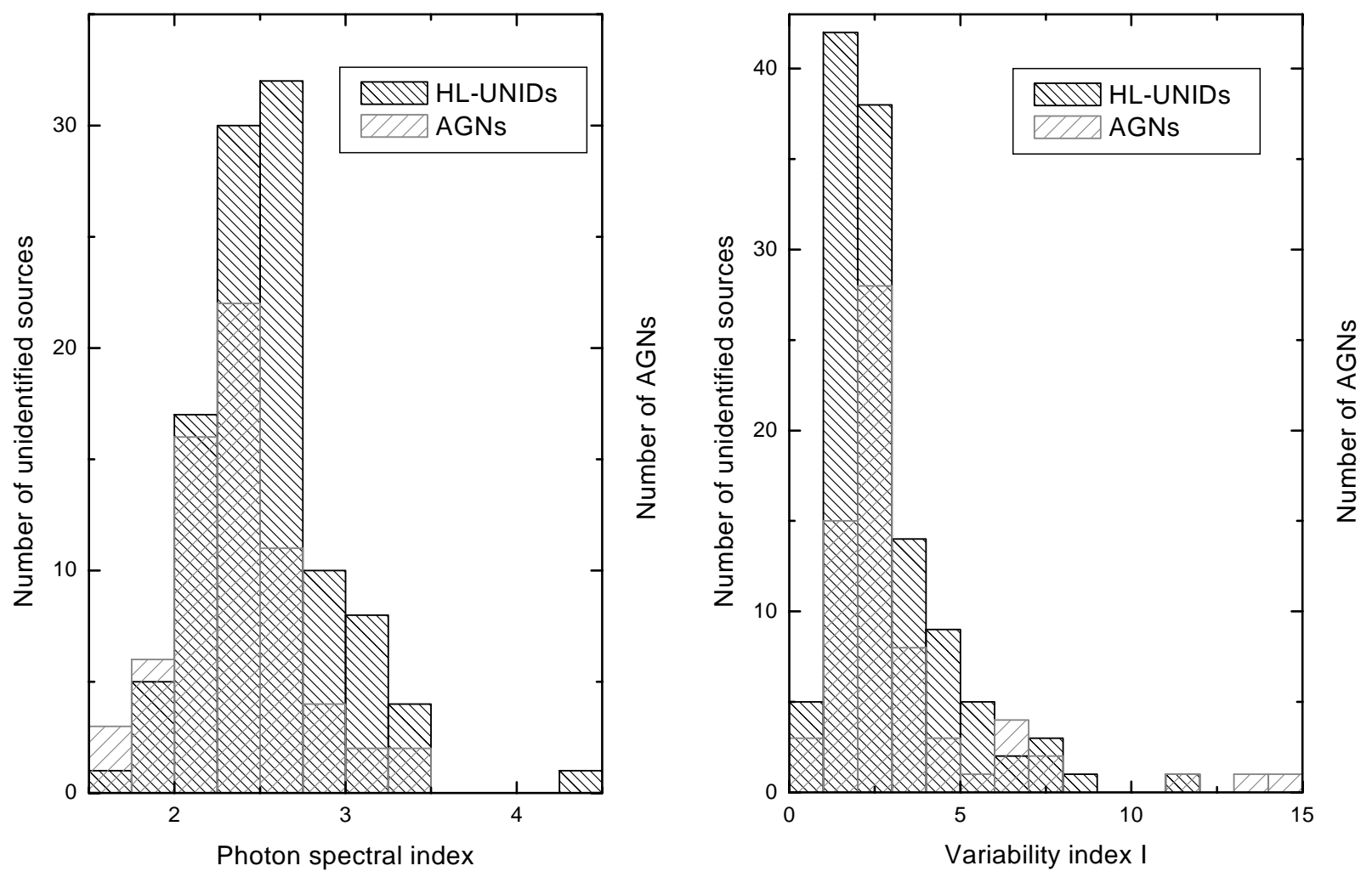

FIG. 1. - Left: Distribution of the photon spectral index of all unidentified sources with Galactic latitudes $|b|>10^{\circ}$. A Gaussian fit would give a mean equal to 2.6 and deviation equal to 0.3 . Most of the sources have steep spectra, which is consistent with the AGN population. Right: Histogram for the variability index $I$ of each of the HL-UnidS ( $I=1$ is the mean value of $I$ for pulsars). A Gaussian curve would have, in this case, a mean at $I=2$, which is a value already $2 \sigma$ above the expected one for a nonvariable population, and a deviation equal to 0.7 . More than half of these sources are classified as likely variables. Taking only those sources having $|b|>40^{\circ}$, both mean values are even higher. See Torres et al. (2001a, 2001b) for details.

particle population giving rise-in the same region-to $\gamma$-rays (through inverse Compton scattering) and optical emission (through synchrotron radiation), then we can also expect gravitational magnification of the optical luminosity. In some cases with high (lensing) optical depth, we could even expect simultaneous intensity variations in both bands, which is something that could in principle be tested with the improved capabilities and source location accuracy of the forthcoming GLAST satellite. Optical monitoring of stellarlike sources inside GLAST error boxes could lead to the identification of new $\gamma$-ray blazars and to some knowledge of their redshift distribution. One interesting fact is that the spectral evolution that we predict below will happen only in the $\gamma$-ray band, since it critically depends on the size of the $\gamma$-ray spheres (given by the opacity to pair production); this process is not operative in the optical band. The idea that some extreme properties of distant AGNs can be the result of gravitational microlensing is not new (e.g., Ostriker \& Vietri 1985; Gopal-Krishna \& Subramanian 1991; Romero, Surpi, \& Vucetich 1995). However, microlensing effects upon the $\gamma$-ray emission have not been discussed in detail yet.

It is usually assumed that the a priori probability of finding a small group of distant, gravitationally magnified objects is below $1 \%$. Indeed, recent results (Wythe \& Turner 2002) that take into account the clustering of lenses in interposed galaxies give values between $10^{-2}$ and $10^{-3}$ for the a priori probability of finding gravitational magnified sources in random directions of the sky. In addition, for those directions where there is gravitational lensing, the probability of it happening with an optical depth above 0.2 is very high. In our case, the number of potential compact $\gamma$-ray-emitting background sources is extremely large: only the last version of the Véron-Cetty \& Véron (2001) catalog — which is still very incomplete at high redshifts - contains more than $10^{3}$ already identified blazars in addition to more than $10^{4}$ quasars and other, less energetic, AGNs. Actually, the enhanced sensitivity of the GLAST mission is expected to uncover more than $10^{4}$

$\gamma$-ray-emitting AGNs (Gehrels \& Michelson 1999). If the actual number of $\gamma$-ray-emitting AGNs below the EGRET detection threshold is, say, $10^{7}$ (which can be a conservative assumption: approximately $1 \gamma$-ray blazar per $10^{4}$ galaxies), they might produce high-latitude sources that lack a clear low-frequency counterpart. Even when considering reduced probabilities for microlensing with large magnifications (i.e., $\sim \tau / A^{2}$, where $\tau$ is the local optical depth and $A$ is the magnification), we still found that a handful of the sources at high latitudes already observed by EGRET, and maybe a few hundred of sources to be observed by GLAST, can be the result of microlensing. For instance, considering a random lensing probability of $5 \times 10^{-3}$ and a local value of $\tau$ of $O(1)$ (even larger values are possible because the presence of superluminal components in blazars; see below) and amplifications of $\sim 100$, we could produce by lensing $\sim 5$ high-latitude sources (as we see below) without counterparts at lower energies. Of course, if a large number of distant blazars are, say, 1 order of magnitude below GLAST sensitivity, in such a way that they do not need huge amplifications in order to be detected, then the number of possible microlens- 
ing cases could very much increase. Assuming an amplification of a factor of 10, the number of cases could increase to several hundreds in the forthcoming large GLAST sample.

\section{MICROLENSING MODEL FOR EXTENDED SOURCES}

We consider a distant and weak $\gamma$-ray-emitting blazar with GeV flux that is well below EGRET sensitivity threshold and with radio flux that is at the mJy level, which is also below the sensitivity of typical all-sky surveys. Assuming a galaxy interposed in the line of sight, the lens equation, in the lens plane, is (Chang \& Refsdal 1979; Schneider, Ehlers, \& Falco 1992)

$$
\boldsymbol{r}-\boldsymbol{r}_{0}-R_{\mathrm{E}}^{2} \frac{\boldsymbol{r}}{r^{2}}-\operatorname{diag}(\kappa+\gamma, \kappa-\gamma) \boldsymbol{r}-\omega_{0}=0,
$$

where the coordinate system is centered on the lens, with the orientation of the orthonormal basis $\left\{\boldsymbol{e}_{1}, \boldsymbol{e}_{2}\right\}$ chosen to diagonalize the quadruple matrix; the source is at $\boldsymbol{r}_{0}$, and the image position is $\boldsymbol{r}$. The fourth and fifth terms in equation (1) arise from the deflection imprinted by the host galaxy as a whole: $\kappa$ and $\gamma$ are the focusing and the shear of the galaxy at the point-mass position, respectively. The only effect of $\omega_{0}$ is to change the unperturbed source position $\boldsymbol{r}_{0}$ by a constant. We shall ignore $\omega_{0}$ and assume a source position $\boldsymbol{s}=\boldsymbol{r}_{0}+\omega_{0}$ in the lens plane. With new appropriate coordinates defined in the lens and the source plane (Schneider et al. 1992), $\boldsymbol{X}=\left[(1-\kappa+\gamma)^{1 / 2} / R_{\mathrm{E}}\right] \boldsymbol{r}$ and $\boldsymbol{Y}=\left[1 / R_{\mathrm{E}}(1-\kappa+\gamma)^{1 / 2}\right] \boldsymbol{s}$, the lens equation becomes $\boldsymbol{Y}=\varepsilon[\operatorname{diag}(\Lambda, 1)] \boldsymbol{X}-\boldsymbol{X} /|\boldsymbol{X}|^{2}$, where $\varepsilon=\operatorname{sgn}(1-\kappa+\gamma)$ and $\Lambda=(1-\kappa-\gamma) /(1-\kappa+\gamma)$. The solution of the latter equation can be found by reducing the problem to a fourthorder equation for $X^{2}$ (Schneider et al. 1992).

We finally obtain the magnification, $A=I_{\mathrm{obs}} / I_{0}$, as

$$
A=\frac{|1-\kappa+\gamma|^{-1}\left(X_{1}^{2}+X_{2}^{2}\right)^{2}}{\left|\Lambda\left(X_{1}^{2}+X_{2}^{2}\right)^{2}+\varepsilon(1-\Lambda)\left(X_{1}^{2}-X_{2}^{2}\right)-1\right|} .
$$

For extended circular sources, the magnification is given by (e.g., Han, Park, \& Jeong 2000)

$$
A=\frac{\int_{0}^{2 \pi} \int_{0}^{r_{\mathrm{s}}} \mathscr{I}(r, \varphi) A_{0}(r, \varphi) r d r d \varphi}{\int_{0}^{2 \pi} \int_{0}^{r_{\mathrm{s}}} \mathscr{I}(r, \varphi) r d r d \varphi},
$$

where $(r, \varphi)$ are polar coordinates in a reference frame placed at the center of the source, $r_{s}$ is the radius of the source, $\mathscr{I}(r, \varphi)$ is the surface intensity distribution of the source, and $A_{0}$ is the sum of the magnifications of all images. We define $R=r / R_{\mathrm{E}}$ and suppose that the lens is moving with constant velocity $v^{4}$

The radius of the internal regions of $\mathrm{AGNs}$ depends on the energy as a power law, $r_{\gamma} \propto E^{p}$ (Blandford \& Levinson

\footnotetext{
${ }^{4}$ We choose the origin of time $(t=0)$ as the instant of closest approach between the lens and the source. Then, if the center of the source is placed at $\boldsymbol{b}=\left(b_{1}, b_{2}\right)$ when $t=0$ (projected on the plane of the lens), then the position of any point of the source with polar coordinates $(r, \varphi)$ is $s_{1}(t)=b_{1}-v t \cos \theta+r \cos \varphi$ and $s_{2}(t)=b_{2}-v t \sin \theta+r \sin \varphi$, where $v=|\boldsymbol{v}|, \theta$ is the angle between $\boldsymbol{v}$ and $\boldsymbol{e}_{1}, 0 \leq r \leq r_{s}$ and $0 \leq \varphi \leq 2 \pi$. In units of the Einstein radius, $Y_{1}=\left[B_{1}-T \cos \theta+R \cos \varphi\right] /(1-\kappa+\gamma)^{1 / 2}$, and $Y_{2}=\left[B_{2}-T \sin \theta+R \sin \varphi\right] /(1-\kappa+\gamma)^{1 / 2}$, where $T=v t / R_{\mathrm{E}}$, and $B_{1,2}=b_{1,2} / R_{\mathrm{E}}$. When $\gamma=0$ (no shear), we can take $\theta=0$ and $\boldsymbol{B}=\left(0, B_{0}\right)$ without losing generality.
}

1995). We then define a reference source with radius $r_{\text {ref }}$ and $\gamma$-ray energy $E_{\text {ref }}$ such that $R_{\gamma}(E)=R_{\text {ref }}\left(E / E_{\text {ref }}\right)^{p}$, where capital letters stand for normalized quantities using the Einstein radius. We assume that the intensity of the source is uniform and that its spectrum follows approximately a power law $I_{0}(E)=I_{\text {ref }}\left(E / E_{\text {ref }}\right)^{-\xi}$, with $\xi \in(1.7,2.7)$, where $I_{\text {ref }}$ is the intensity of the reference source (Krolik 1999). The surface intensity distribution of the source will then be $\mathscr{I}_{0}(E)=I_{0}(E) / \pi R_{\gamma}(E)^{2}$, from which the magnification can be computed.

Using that $A=I / I_{0}$, we define $J \equiv I / I_{\text {ref }}=A\left(E / E_{\text {ref }}\right)^{-\xi}$, which is the intensity in units of $I_{\text {ref }}$. We adopt $p=1.1$, $\xi=2$, and a reference source with dimensionless radius $R_{\text {ref }}=2 \times 10^{-3}$ and $\gamma$-ray energy $E_{\text {ref }}=0.1 \mathrm{GeV}$ (Blandford \& Levinson 1995). In Figure 2 we show just an example of the results of our numerical computations. The $\gamma$-ray emission of the background AGN can be amplified significantly. This magnification can make an otherwise unnoticed source exceed the detection threshold. Note also that the $\gamma$ sphere corresponding to $10 \mathrm{GeV}$, whose size is similar to the optical emitting regions of some typical AGNs, is negligibly amplified, while the lower energy curves - well within the EGRET range - all show magnifications in excess of a factor of 100. This phenomenon also has a particular spectral signature, which is produced by the differential amplification of the different $\gamma$-regions. The spectral evolution (chromaticity) effect is a change in the spectral slope at medium energies. This break, predicted only for microlensing events, and its peculiar time evolution - it shifts toward high energies as times goes by since transit - can be used as a specific test to differentiate this from other phenomena (see Fig. 2, right panels).

\section{TIMESCALES AND NUMBER OF EVENTS}

The timescale of lensing variability is $t_{0}=R_{\mathrm{E}} / v$. For instance: for $M=0.1 M_{\odot}, H_{0}=75 \mathrm{~km} \mathrm{~s}^{-1} \mathrm{Mpc}^{-1}, z_{s}=1$, $z_{l}=0.1, \Omega_{0}=0.2$, and $v=5000 \mathrm{~km} \mathrm{~s}^{-1}, t_{0} \sim 170$ days, while the half-width of the peaks shown in Figure 2 is about 9 days. This may result in a variable source for separated EGRET viewing periods (typically of $\sim 15$ days each). If, instead, $M_{l} \sim 5 M_{\odot}$ and $v \sim 1000 \mathrm{~km} \mathrm{~s}^{-1}$, then the halfwidth of the peaks is $\sim 1000$ days, and the $\gamma$-ray source would most likely be seen as a steady, nonvariable detection. ${ }^{5}$

Following Subramanian \& Gopal-Krishna (1991), if the lenses have the same mass and the observing period is $\Delta t$, then the expected number of events, in the case of a background source moving with velocity $v$, will be

$$
N=\tau\left(1+\frac{2 \Delta t}{\pi u t_{0}}\right)
$$

where $\tau$ is the optical depth and $u=b / r_{\mathrm{E}}=B / R_{\mathrm{E}}$ is usually taken as 1 . The second term in parentheses corresponds to the increment of the probability of detecting a microlensing event due to the movement of the source (see Romero et al.

\footnotetext{
${ }^{5}$ The velocities chosen are typical for extragalactic objects. For galaxies at redshifts $z \sim 0.1$ or more, their recession velocities very much exceed the one that we are using (by more than 1 order of magnitude). The latter are relative velocities involving small traversal components of the recession speed and proper motions of the star within the galaxy and the galaxy itself (with respect to its cluster). A worked example (for BL Lac 0846+51) can be found in Nottale (1986), for which he quotes $300<v<3000 \mathrm{~km} \mathrm{~s}^{-1}$.
} 

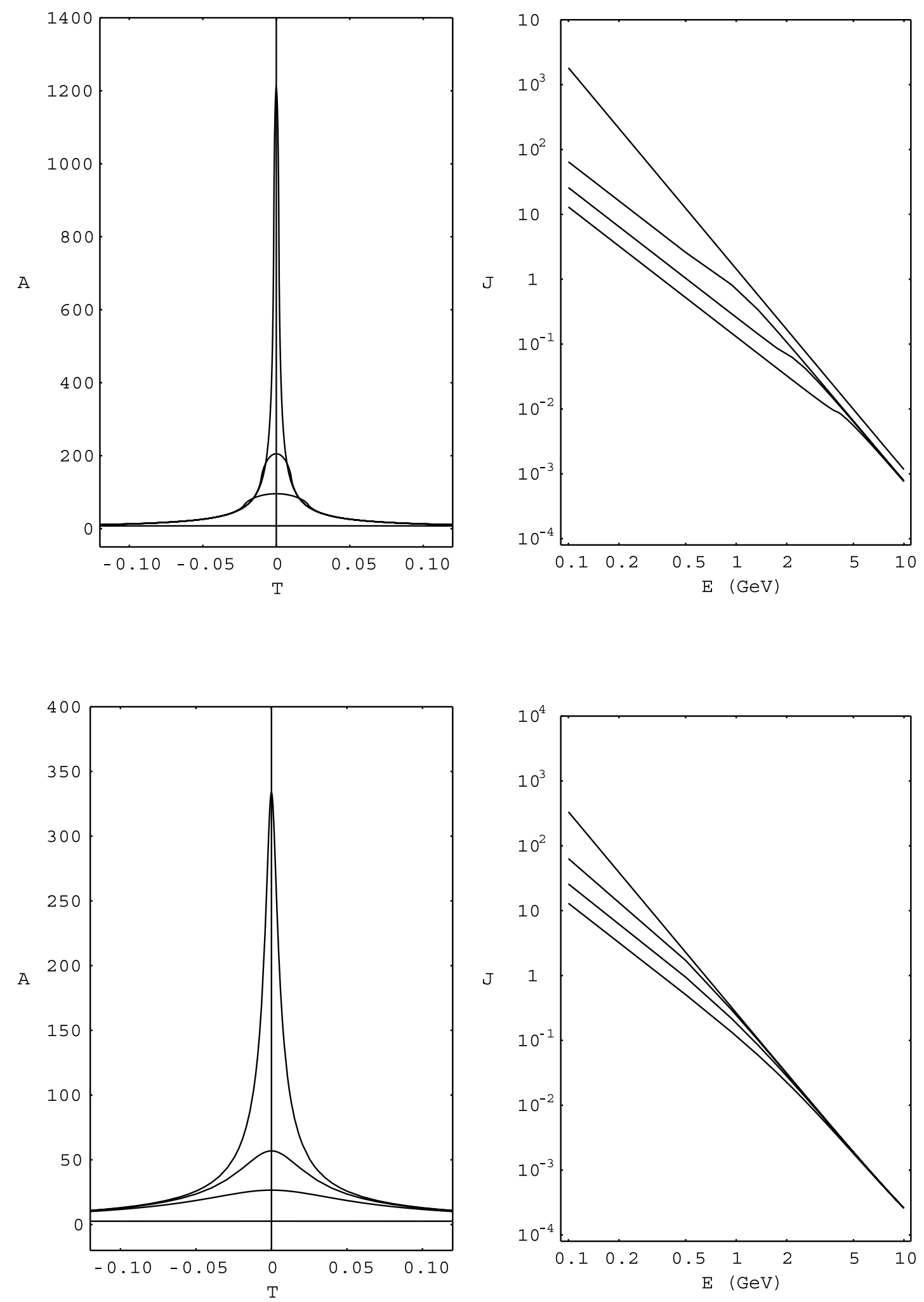

FIG. 2.-Lensing results for a dimensionless impact parameter equal to $B / R_{s}=b / r_{s}=0.5$ (upper panel) and 2 (lower panel). Notice the specific feature (a

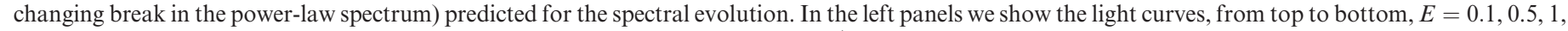

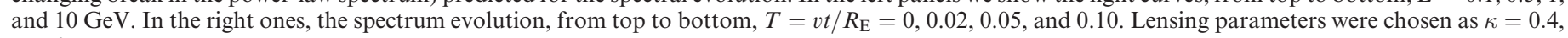
$\gamma=0$.

1995; Surpi, Romero, \& Vucetich 1996). The optical depth can be defined as the ratio of the surface mass density of microlensing matter to the critical mass density $\Sigma_{\text {crit }}=c^{2} D_{o s} / 4 \pi G D_{o l} D_{l s}$. For simplicity, we shall assume that the lenses are located at a distance from the center of the galaxy much smaller than the core radius. Then, $\kappa \sim \Sigma_{c} / \Sigma_{\text {crit }}$, with $\Sigma_{c}$ as the central surface density. The high surface mass density associated with the core of normal 
galaxies, along with the usual assumption that most of this mass is in the form of compact objects, naturally leads to high optical depths for microlensing. For instance, in the case of the lensed quasar Q2237-031, where four images are well resolved, lensing models indicate values of $\tau \sim 0.5$ (Schneider et al. 1988), which are corroborated by the detections of microlensing-based optical variability with relatively high duty cycles (e.g., Corrigan et al. 1991). Other lensed sources display even higher duty cycles (e.g., Koopmans \& de Bruyn 2000).

For a mass distribution of lenses given by $N(M) \propto M^{-\alpha}$ for $M_{\min } \leq M \leq M_{\max }$ (Salpeter 1955), the number of expected microlensing events by stars with masses in the range $\left(M_{1}, M_{2}\right)$, included in the total mass range $\left(M_{\min }, M_{\max }\right)$, during $\Delta t$ days of observations is

$$
N_{M_{1}-M_{2}}^{\Delta t}=2.16 \sqrt{\kappa \tau} \frac{v}{c} \frac{D\left(\alpha, M_{1}, M_{2}\right)}{B\left(\alpha, M_{\min }, M_{\max }\right)} \frac{\Delta t}{30 \text { days }},
$$

where the functions $B$ and $D$ are, respectively,

$$
\begin{aligned}
& B\left(\alpha, M_{\min }, M_{\max }\right)= \\
& \begin{cases}{\left[\left(\frac{M_{\max }}{M_{\odot}}\right)^{2-\alpha}-\left(\frac{M_{\min }}{M_{\odot}}\right)^{2-\alpha}\right] /(2-\alpha)} & \alpha \neq 2, \\
\ln \left(\frac{M_{\max }}{M_{\min }}\right) & \alpha=2,\end{cases}
\end{aligned}
$$

and

$$
\begin{aligned}
& D\left(\alpha, M_{1}, M_{2}\right)= \\
& \begin{cases}2\left[\left(\frac{M_{2}}{M_{\odot}}\right)^{(3 / 2)-\alpha}-\left(\frac{M_{1}}{M_{\odot}}\right)^{(3 / 2)-\alpha}\right] /(3-2 \alpha) & \alpha \neq \frac{3}{2}, \\
\ln \left(\frac{M_{2}}{M_{1}}\right) & \alpha=\frac{3}{2},\end{cases}
\end{aligned}
$$

and we have assumed a case with $z_{s} \sim 0.9$ and $z_{l} \sim 0.1$ to fix the numerical coefficient. Clearly, the total number of events will strongly depend (apart from the expected influence of $\tau$ and $\kappa$ ) on the velocity of the source and the index $\alpha$, which is usually taken in the range $(2,3)$.

A particularly interesting case of source candidates is blazars in which the high-energy emission is produced in a superluminal component with apparent velocity $v>c$ in the lens plane (Gopal-Krishna \& Subramanian 1991). They will produce $\gamma$-ray sources with the highest levels of variability. We find that during the EGRET lifetime, hundreds of events can be expected for optical depths in the range 0.2 0.4 . Even for values of $\tau$ as low as $10^{-3}$, if the source is apparently superluminal, then the number of expected events in the EGRET observing time is above 10. Instead, sources whose velocities in the lens plane are much smaller than $c$ (say, about $5000 \mathrm{~km} \mathrm{~s}^{-1}$ ) will produce only a few events. A complete study of the distribution of lens masses within this model will be presented elsewhere.

\section{CONCLUDING REMARKS}

In summary, we have found that gravitational microlensing of the innermost regions of distant AGNs can produce unidentified sources that are compatible with those observed at high Galactic latitudes. While large amplification factors are obtained for $\gamma$-rays, a negligible magnification results in the radio band. In the case of the optical emission, if it is the same particle population giving rise to $\gamma$-rays and optical emission, then we can also expect magnification of the optical luminosity. The $\gamma$-ray spectral evolution provides a specific signature for the microlensing events that can be used to differentiate this from other kind of phenomena. Higher (lensing) optical depth, or the presence of shear, will lead to a diversity of light curves. We remark that we have used the Chang \& Refsdal (1979) model as the gravitational lensing scenario but that galaxies with denser cores would require a more detailed treatment. This would necessarily include the study of caustic patterns of all stars at a time in order to get the light curves. We shall explore the lensing model in much more detail in a subsequent publication (E. Eiroa et al. 2002, in preparation).

This work has been partially supported by UBA (UBACYT X-143, E. F. E.), CONICET (D. F. T., and PIP 0430/ 98, G. E. R.), ANPCT (PICT 98 03-04881, G. E. R.), and Fundación Antorchas (separate grants to G. E. R. and D. F. T.). G. E. R. thanks the kind support from the astrophysics group at the MPIfK, Heidelberg, where part of his research was carried out. E. L. Turner is acknowledged for providing his - at that time - unpublished results and for useful discussions. R. C. Hartman, D. J. Thompson, and S. Ritz are acknowledged for comments and discussions. We are especially grateful to R. C. Hartman for his insightful remarks.
Blandford, R. D., \& Levinson, A. 1995, ApJ, 441, 79

Chang, K., \& Refsdal, S. 1979, Nature, 282, 561

Corrigan, R. T., et al. 1991, AJ, 102, 34

Gehrels, N., Macomb, D. J., Bertsch, D. L., Thompson, D. J., \& Hartman, R. C. 2000 , Nature, 404, 363

Gehrels, N., \& Michelson, P. 1999, Astropart. Phys., 11, 277

Gopal-Krishna, \& Subramanian, K. 1991, Nature, 349, 766

Grenier, I. 2000, A\&A, 364, L93

Han, C., Park, S-H., \& Jeong, J-H. 2000, MNRAS, 316, 97

Hartman, R. C., et al. 1999, ApJS, 123, 79

Koopmans, L. V. E., \& de Bruyn, A. G. 2000, A\&A, 358, 793

Krolik, J. H. 1999, Active Galactic Nuclei (Princeton: Princeton Univ. Press)

Mattox, J. R., Schachter, J., Molnar, L., Hartman, R. C., \& Patnaik, A. R. 1997, ApJ, 481, 95

Nottale, L. 1986, A\&A, 157, 383

Ostriker, J., \& Vietri, M. 1985, Nature, 318, 446

Özel, M. E., \& Thompson, D. J. 1996, ApJ, 463, 105

Reimer, O., \& Bertsch, D. L. 2001, Proc. 27th Int. Cosmic-Ray Conf. (Hamburg), 2566

\section{REFERENCES}

Romero, G. E. 2001, in The Nature of Unidentified Galactic HighEnergy Gamma-Ray Sources, ed. A. Carramiñana, O. Reimer, \& D. J. Thompson (Dordrecht: Kluwer), 65

Romero, G. E., Surpi, G., \& Vucetich, H. 1995, A\&A, 301, 641

Salpeter, E. E. 1955, ApJ, 121, 161

Schneider, D., et al. 1988, AJ, 95, 1619

Schneider, P., Ehlers, J., \& Falco, E. E. 1992, Gravitational Lenses (Berlin: Springer)

Subramanian, K., \& Gopal-Krishna, 1991, A\&A, 248, 55

Surpi, G., Romero, G. E., \& Vucetich, H. 1996, Rev. Mexicana Astron. Astrofis. 32, 153

Torres, D. F., Pessah, M. E., \& Romero, G. E. 2001b, Astron. Nachr., 322, 223

Torres, D. F., Romero, G. E., Combi, J., Benaglia, P., Punsly, B., \& Andernach, H. 2001a, A\&A, 370, 468

Totani, T., \& Kitayama, T. 2000, ApJ, 545, 572

Véron-Cetty, M.-P., \& Véron, P. 2001, A\&A, 374, 92

Wythe, J. S. B., \& Turner, E. L. 2002, ApJ, 567, 18 